04,02

\title{
Сверхтонкие взаимодействия в узлах меди антиферромагнитных соединений, аналогов сверхпроводящих металлооксидов меди
}

\author{
(С) Е.И. Теруков ${ }^{1,2}$, А.В. Марченко ${ }^{3}$, Ф.С. Насрединов ${ }^{4}$, А.А. Левин ${ }^{1}$, А.А. Лужков ${ }^{3}$, П.П. Серегин ${ }^{3, \text { Ф }}$ \\ ${ }^{1}$ Физико-технический институт им. А.Ф. Иофффе РАН, \\ Санкт-Петербург, Россия \\ ${ }^{2}$ Санкт-Петербургский государственный электротехнический университет „ЛЭТИ“ им. В.И. Ульянова (Ленина), \\ Санкт-Петербург, Россия \\ ${ }^{3}$ Российский государственный педагогический университет им. А.И. Герцена, \\ Санкт-Петербург, Россия \\ ${ }^{4}$ Санкт-Петербургский политехнический университет Петра Великого, \\ Санкт-Петербург, Россия \\ ๑ E-mail: ppseregin@mail.ru
}

Поступила в Редакцию 14 октября 2019 г.

В окончательной редакции 14 октября 2019 г.

Принята к публикации 29 октября 2019 г.

\begin{abstract}
Эмиссионные мёссбауэровские спектры ${ }^{61} \mathrm{Cu}\left({ }^{61} \mathrm{Ni}\right)$ диэлектрических металлоксидов двухвалентной меди $\mathrm{Ca}_{1-x} \mathrm{Sr}_{x} \mathrm{CuO}_{2}, \mathrm{Ca}_{2} \mathrm{CuO}_{2} \mathrm{Cl}_{2}, \mathrm{SrCuO}_{2}, \mathrm{Sr}_{2} \mathrm{CuO}_{2} \mathrm{Cl}_{2}, \mathrm{YBa}_{2} \mathrm{Cu}_{3} \mathrm{O}_{7-x}, \mathrm{La}_{2-x} \mathrm{Sr}_{x} \mathrm{CuO}_{4}, \mathrm{Nd}_{2-x} \mathrm{Ce}_{x} \mathrm{CuO}_{4}$ отвечают квадрупольному и зеемановском взаимодействию ядер ${ }^{61} \mathrm{Ni}$ с локальными полями в узлах меди, тогда как для сверхпроводящих металлоксидов спектры соответствуют взаимодействию квадрупольного момента ядер ${ }^{61} \mathrm{Ni} \mathrm{c}$ тензором градиента электрического поля (ГЭП). Для обеих групп металлоксидов наблюдаются линейные зависимости постоянных квадрупольного взаимодействия как на ядрах ${ }^{61} \mathrm{Ni}$ (данные эмиссионной мессбауэровской спектроскопии на изотопах ${ }^{61} \mathrm{Cu}\left({ }^{61} \mathrm{Ni}\right)$ ), так и на ядрах ${ }^{63} \mathrm{Cu}$ (данные ядерного магнитного резонанса на изотопе ${ }^{63} \mathrm{Cu}$ ) от расчетных значений главной компоненты тензора решеточного ГЭП в узлах меди. Этот факт объясняется неизменными значениями валентной составляющей ГЭП, как для зонда ${ }^{61} \mathrm{Ni}^{2+}$, так и для зонда ${ }^{63} \mathrm{Cu}^{2+}$ во всех металлоксидах двухвалентной меди.
\end{abstract}

Ключевые слова: антиферромагнетики, высокотемпературные сверхпроводники, эмиссионная мессбауэровская спектроскопия, ЯМР, тензор градиента электрического поля.

DOI: 10.21883 /FTT.2020.03.49000.606

\section{1. Введение}

Многие сверхпроводящие металлоксиды двухвалентной меди (высокотемпературные сверхпроводники, ВТСП) были получена из своих аналогов - антиферромагнитных диэлектрических соединений, таких как $\mathrm{YBa}_{2} \mathrm{Cu}_{3} \mathrm{O}_{6}$ [1], $\mathrm{La}_{2} \mathrm{CuO}_{4}$ [2], $\mathrm{Nd}_{2} \mathrm{CuO}_{4}$ [3], $\mathrm{CaCuO}_{2}$ [4], $\mathrm{SrCuO}_{2}$ [5], $\mathrm{Ca}_{2} \mathrm{CuO}_{2} \mathrm{Cl}_{2}$ [6] и $\mathrm{Sr}_{2} \mathrm{CuO}_{2} \mathrm{Cl}_{2}$ [7], либо методом гетеровалентного замещения редкоземельных металлов, либо методом изменения степени окисления атомов меди. Все эти соединения представляют интерес как в плане модельных систем с анизотропным взаимодействием, так и в плане изучения возможной связи между магнетизмом и сверхпроводимостью.

Мёссбауэровская спектроскопия на различных изотопах $\left({ }^{57} \mathrm{Fe},{ }^{119} \mathrm{Sn},{ }^{155} \mathrm{Gd},{ }^{161} \mathrm{Dy},{ }^{170} \mathrm{Yb}\right)$ широко используется для изучения ВТСП на основе металлоксидов меди [8]. Наибольший интерес такие исследования представляют, если мёссбауэровский зонд находится в узлах меди. В частности, как было показано в [9], для исследования комбинированного магнитного и электрического квадрупольного взаимодействия в узлах меди решеток ВТСП целесообразно использовать эмиссионный вариант мёссбауэровской спектроскопии на изотопах
${ }^{61} \mathrm{Cu}\left({ }^{61} \mathrm{Ni}\right)$. В этом случае мёссбауэровский зонд ${ }^{61} \mathrm{Ni}^{2+}$, образующийся после радиоактивного распада ${ }^{61} \mathrm{Cu}$, оказывается в медных узлах, а ядерные и атомные параметры зонда наиболее удобны для определения параметров комбинированного сверхтонкого взаимодействия в узлах меди. В настоящей работе метод эмиссионной мёссбауэровской спектроскопии на изотопах ${ }^{61} \mathrm{Cu}\left({ }^{61} \mathrm{Ni}\right)$ используется для исследования сверхпроводящих и диэлектричских соединений $\mathrm{Ca}_{1-x} \mathrm{Sr}_{x} \mathrm{CuO}_{2}, \mathrm{Ca}_{2} \mathrm{CuO}_{2} \mathrm{Cl}_{2}$, $\mathrm{SrCuO}_{2}, \mathrm{Sr}_{2} \mathrm{CuO}_{2} \mathrm{Cl}_{2}, \mathrm{YBa}_{2} \mathrm{Cu}_{3} \mathrm{O}_{7-x}, \mathrm{La}_{2-x} \mathrm{Sr}_{x} \mathrm{CuO}_{4}$ и $\mathrm{Nd}_{2-x} \mathrm{Ce}_{x} \mathrm{CuO}_{4}$.

\section{2. Методика эксперимента}

Поликристаллические образцы $\mathrm{Ca}_{1-x} \mathrm{Sr}_{x} \mathrm{CuO}_{2}$, $\mathrm{Ca}_{2} \mathrm{CuO}_{2} \mathrm{Cl}_{2}, \quad \mathrm{SrCuO}_{2}, \quad \mathrm{Sr}_{2} \mathrm{CuO}_{2} \mathrm{Cl}_{2}, \quad \mathrm{YBa}_{2} \mathrm{Cu}_{3} \mathrm{O}_{7-x}$, $\mathrm{La}_{2-x} \mathrm{Sr}_{x} \mathrm{CuO}_{4}$ и $\mathrm{Nd}_{2-x} \mathrm{Ce}_{x} \mathrm{CuO}_{4}$ готовились по методикам, описанным в [1-9]. Однофазность образцов контролировалась рентгеноструктурным анализом. Критические температуры для сверхпроводящих образцов $\mathrm{YBa}_{2} \mathrm{Cu}_{3} \mathrm{O}_{6.9}, \mathrm{La}_{1.85} \mathrm{Sr}_{0.15} \mathrm{CuO}_{4}$ и $\mathrm{Nd}_{1.85} \mathrm{Ce}_{0.15} \mathrm{CuO}_{4}$ были 78,37 и $22 \mathrm{~K}$ соответственно.

Изотоп ${ }^{61} \mathrm{Cu}$ получали по реакции ${ }^{61} \mathrm{Ni}(p, n){ }^{61} \mathrm{Cu}$ с последующим хроматографическим выделением безно- 
сительного препарата ${ }^{61} \mathrm{CuCl}_{2}$. Мёссбауэровские источники на основе металлоксидов меди готовились методом диффузии изотопа ${ }^{61} \mathrm{Cu}$ в готовые керамики при температурах $500-650^{\circ} \mathrm{C}$ в течение $2 \mathrm{~h}$ в атмосфере кислорода [9]. Активность препарата ${ }^{61} \mathrm{Cu}$ и малое время жизни $(\sim 4.5$ h) не позволяли получить мёссбауэровский спектр требуемого качества от одного образца, и для регистрации одного спектра использовалось от 4 до 6 образцов.

Эмиссионные мёссбауэровские спектры ${ }^{61} \mathrm{Cu}\left({ }^{61} \mathrm{Ni}\right)$ снимались на спектрометре SM 4201 TerLab. Стандартным поглотителем служил сплав $\mathrm{Ni}_{0.86} \mathrm{~V}_{0.14}$ (поверхностная плотность $1500 \mathrm{mg} / \mathrm{cm}^{2}$ ). Все спектры снимались при $80 \mathrm{~K}$.

\section{3. Экспериментальные результаты и их обсуждение}

Экспериментальные мёссбауэровские спектры ${ }^{61} \mathrm{Cu}\left({ }^{61} \mathrm{Ni}\right)$ исследованных соединений представляют собой плохо разрешенные мультиплеты (см. рис. 1).

В спектрах сверхпроводящих соединений $\mathrm{YBa}_{2} \mathrm{Cu}_{3} \mathrm{O}_{6.9}, \mathrm{La}_{1.85} \mathrm{Sr}_{0.15} \mathrm{CuO}_{4}$ и $\mathrm{Nd}_{1.85} \mathrm{Ce}_{0.15} \mathrm{CuO}_{4}$ следует ожидать результатов взаимодействия квадрупольного момента ядер ${ }^{61} \mathrm{Ni}$ с тензором градиента электрического поля (ГЭП) в узлах меди. При этом основной уровень ядра ${ }^{61} \mathrm{Ni}$ расщепляется на два (спин $I=3 / 2$, квадрупольный момент $\left.Q_{g r}^{61}=0.162 b[8]\right)$, а возбужденный -

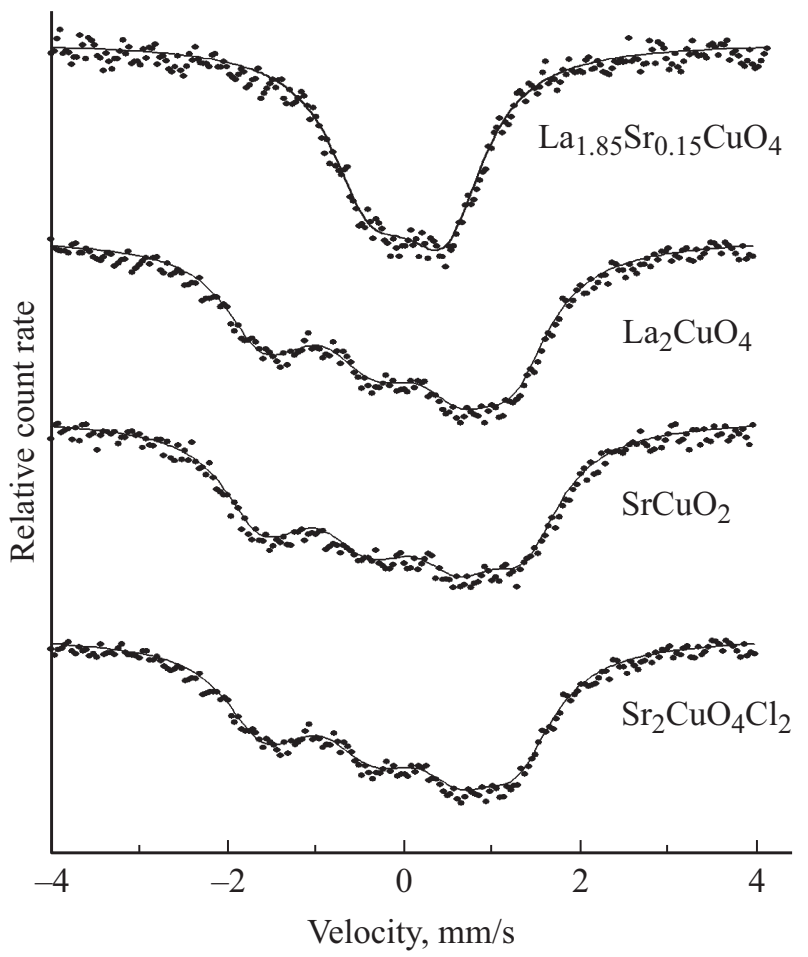

Рис. 1. Эмиссионные мёссбауэровские спектры ${ }^{61} \mathrm{Cu}\left({ }^{61} \mathrm{Ni}\right)$ сверхпроводящей керамики $\mathrm{La}_{1.85} \mathrm{Sr}_{0.15} \mathrm{CuO}_{4}$ и диэлектрических керамик $\mathrm{La}_{2} \mathrm{CuO}_{4}, \mathrm{SrCuO}_{2}$ и $\mathrm{Sr}_{2} \mathrm{CuO}_{2} \mathrm{Cl}_{2}$. на три подуровня $\left(I_{e x}=5 / 2, Q_{e x}^{61}=-0.2 b[8]\right)$. В итоге экспериментальные спектры описывались как наложение пяти линий с отношением интенсивностей 10:4:1:6:9.

Полученный на предварительном этапе исследования спектр примесных атомов ${ }^{61} \mathrm{Cu}\left({ }^{61} \mathrm{Ni}\right)$ в $\mathrm{MgO}$ представлял собой одиночную линию (на рис. 1 не показана) с шириной на полувысоте $G=1.00(2) \mathrm{mm} / \mathrm{s}$, которая несколько превышает удвоенную естественную ширину ядерного уровня ${ }^{61} \mathrm{Ni}\left(2 G_{n a t}=0.78 \mathrm{~mm} / \mathrm{s}\right)$. Уширение объясняется конечной поверхностной плотностью поглотителя, в дальнейшем при обработке экспериментальных мёссбауэровских спектров величина $1 \mathrm{~mm} / \mathrm{s}$ была принята за фиксированную аппаратурную ширину спектральной линии.

Поскольку диэлектрические соединения $\mathrm{Ca}_{1-x} \mathrm{Sr}_{x} \mathrm{CuO}_{2}, \quad \mathrm{Ca}_{2} \mathrm{CuO}_{2} \mathrm{Cl}_{2}, \quad \mathrm{SrCuO}_{2}, \quad \mathrm{Sr}_{2} \mathrm{CuO}_{2} \mathrm{Cl}_{2}$, $\mathrm{YBa}_{2} \mathrm{Cu}_{3} \mathrm{O}_{6}, \mathrm{La}_{2} \mathrm{CuO}_{4}$ и $\mathrm{Nd}_{2} \mathrm{CuO}_{4}$ являются антиферромагнетиками с высокими значениями температуры Нееля [1-9], то структуру их мёссбауэровских спектров в области температур < $200 \mathrm{~K}$ следует рассматривать как результат комбинированного квадрупольного и зеемановского взаимодействия ядер ${ }^{61} \mathrm{Ni}$ с локальными полями. На полученных спектрах это обнаруживается как существенное расширение диапазона скоростей, в котором регистрируются спектральные линии. Для чисто зеемановского взаимодействия мёссбауэровский спектр должен симметрично расщепляться на 12 линий с отношением интенсивностей $10: 4: 1: 6: 6: 3: 3: 6: 6: 1: 4: 10$. В случае комбинированного магнитного и электрического взаимодействия собственные значения гамильтониана для каждого, основного и возбужденного, уровня могут быть найдены из соотношения

$$
\begin{aligned}
E_{m}^{I}= & m g H+\left[e Q U_{z z} / 4 I(2 I-1)\right]\left[3 m^{2}-I(I+1)\right] \\
& \times\left[\left(3 \cos ^{2} \theta-l\right) / 2\right] .
\end{aligned}
$$

Здесь $I-$ спин ядра; $H-$ магнитное поле на ядре; $U_{z z}$ - главная компонента тензора ГЭП на ядре; $\theta-$ угол между главной осью тензора ГЭП и направлением магнитного поля; $m$ - магнитное квантовое число; $g$ - гидромагнитное отношение (для ядра ${ }^{61} \mathrm{Ni}$ в основном состоянии $g=-0.070083 \mathrm{~mm} / \mathrm{s} \cdot \mathrm{T}$ и в возбужденном состоянии $g_{e x}=0.0268 \mathrm{~mm} / \mathrm{s} \cdot \mathrm{T}$ [9]). Симметричное расположение линий спектра при этом нарушается.

Расчетный спектр подгонялся к экспериментальному спектру методом наименыших квадратов. Подгоночными параметрами были не положения отдельных линий, а параметры гамильтониана $H$ и $U_{z z}\left[\left(3 \cos ^{2} \theta-1\right) / 2\right]$, общие для обоих ядерных уровней, а также интенсивности линий. При этом величина множителя $\left(3 \cos ^{2} \theta-1\right) / 2$ определялась с использованием данных [9-16]. Ширины линий были приняты равными $1 \mathrm{~mm} / \mathrm{s}$, как описано выше. Поскольку в спектрах ${ }^{61} \mathrm{Ni}$ диапазон наблюдавшихся изомерных сдвигов «G, мы следили за тем, чтобы центр тяжести расчетного мультиплета не отклонялся от нулевой скорости больше, чем на $0.05 \mathrm{~mm} / \mathrm{s}$. Согласие 


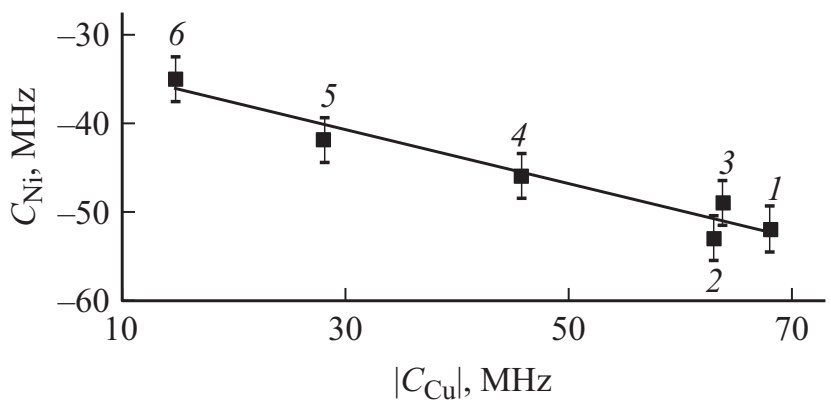

Pис. 2. Соотношение констант квадрупольного взаимодействия $C_{\mathrm{Ni}}$ для центров ${ }^{61} \mathrm{Ni}$ в узлах меди и $C_{\mathrm{Cu}}$ для центров ${ }^{63} \mathrm{Cu}$ в тех же узлах. Обозначения узлов меди в решетках: $1-\mathrm{La}_{1.85} \mathrm{Sr}_{0.15} \mathrm{CuO}_{4}, 2-\mathrm{Cu}(2)$ в $\mathrm{YBa}_{2} \mathrm{Cu}_{3} \mathrm{O}_{7}, 3-\mathrm{La}_{2} \mathrm{CuO}_{4}$, $4-\mathrm{Cu}(2)$ в $\mathrm{YBa}_{2} \mathrm{Cu}_{3} \mathrm{O}_{6}, 5-\mathrm{Nd}_{2} \mathrm{CuO}_{4}, 6-\mathrm{SrCuO}_{2}$.

расчетного и экспериментального спектров оценивалось по критерию $\chi^{2}$.

Результаты обработки мёссбауэровских спектров представлены в виде константы квадрупольного взаимодействия $C_{\mathrm{Ni}}=e Q_{g r}^{61} U_{z z}$ основного состояния ${ }^{61} \mathrm{Ni}$ (для возбужденного состояния можно пересчитать через отношение квадрупольных моментов) и индукции эффективного магнитного поля на ядре $H$. Значимые корреляции между полученными значениями $H$ и $C$ обнаружены не были. Во всех изученных антиферромагнитных соединений величины $H$ были в пределах от 8.5 T до $10.0 \mathrm{~T}$.

При интерпретации величин $C_{\mathrm{Ni}}$ мы использовали известные значения константы квадрупольного взаимодействия $C_{\mathrm{Cu}}=e Q^{63} U_{z z}$ для центров ${ }^{63} \mathrm{Cu}$, полученные для тех же материалов методом ядерного магнитного резонанса (ЯМР) [14-16] (здесь $Q^{63}$ - квадрупольный момент ядра $\left.{ }^{63} \mathrm{Cu}\right)$. На рис. 2 показана корреляция между $C_{\mathrm{Ni}}$ и $\left|C_{\mathrm{Cu}}\right|$ в узлах меди металлоксидов меди (малое значение спина ${ }^{63} \mathrm{Cu}$ не позволяет определить знак $C_{\mathrm{Cu}}$, и поэтому она указана по абсолютной величине).

Точки на рис. 2 удовлетворительно укладываются на прямую

$$
C_{\mathrm{Ni}}=-0.31\left|C_{\mathrm{Cu}}\right|-32
$$

(здесь и далее значения констант квадрупольного взаимодействия приводятся в $\mathrm{MHz}$ ).

Наличие корреляции между данными для $C_{\mathrm{Ni}}$ и $C_{\mathrm{Cu}}$ указывает на возможность их интерпретации с единой точки зрения. Для этой цели нами были проведены расчеты параметров тензора решеточного ГЭП на узлах меди во всех рассмотренных материалах.

Для обоих зондов ${ }^{61} \mathrm{Ni} \mathrm{и}{ }^{63} \mathrm{Cu}$ ГЭП на ядре создается ионами кристаллической решетки (решеточный ГЭП) и несферической валентной оболочкой атома-зонда (валентный ГЭП)

$$
e Q U_{z z}=e Q(1-\gamma) V_{z z}+e Q(1-R) W_{z z},
$$

где $Q$ - квадрупольный момент атомного ядра, $U_{z z}-$ главная компонента тензора суммарного ГЭП, $V_{z z}$ и
$W_{z z}-z$-компоненты тензоров решеточного и валентного ГЭП в рассматриваемом узле, а $\gamma$ и $R-$ коэффициенты Штернхеймера для этого зонда.

Расчет тензора решеточного ГЭП проводился в рамках модели точечных зарядов и для этих расчетов необходимо лишь знание параметров элементарной ячейки соответствующего кристалла (эти данные для исследованных соединений известны [10-13]) и зарядов во всех узлах решетки. Компоненты тензора ГЭП определялись по формулам

$$
\begin{gathered}
V_{p p}=\sum_{k} e_{k}^{*} \sum_{i} \frac{1}{r_{k i}^{3}}\left(\frac{3 p_{k i}^{2}}{r_{k i}^{2}}-1\right)=\sum_{k} e_{k}^{*} G_{p p k}, \\
V_{p q}=\sum_{k} e_{k}^{*} \sum_{i} \frac{3 p_{k i} q_{k i}}{r_{k i}^{5}}=\sum_{k} e_{k}^{*} G_{p q k},
\end{gathered}
$$

где $k$ - индекс суммирования по подрешеткам, $i$ индекс суммирования по узлам подрешетки, $q, p-$ декартовы координаты, $e_{k}^{*}$ - заряды атомов $k$-подрешетки, $r_{k i}$ - расстояние от $k i$-иона до рассматриваемого узла. Решеточные суммы $G_{p p k}$ и $G_{p q k}$ подсчитывались на ЭВМ, суммирование проводилось внутри сферы радиуса $30 \AA$ (больший радиус суммирования не давал изменения в результатах).

При расчетах $V_{z z}$ принципиальное значение имеет выбор модели зарядового распределения по узлам решетки. Для диэлектрических оксидов заряды соответствующих атомов считались равными их традиционной валентности:

$$
\begin{aligned}
& \mathrm{Ca}_{1-x}^{2-} \mathrm{Sr}^{2+} \mathrm{Cu}^{2+} \mathrm{O}_{2}^{2-}, \quad \mathrm{Ca}_{2}^{2+} \mathrm{Cu}^{2+} \mathrm{O}_{2}^{2-} \mathrm{Cl}_{2}^{-}, \quad \mathrm{Sr}^{2+} \mathrm{Cu}^{2+} \mathrm{O}_{2}^{2-} \text {, } \\
& \mathrm{Sr}_{2}^{2+} \mathrm{Cu}^{2+} \mathrm{O}_{2}^{2-} \mathrm{Cl}_{2}^{-} \mathrm{Cu}^{2+} \mathrm{O}^{2-}, \mathrm{Y}^{3+} \mathrm{Ba}_{2}^{2+} \mathrm{Cu}(1)^{+} \mathrm{Cu}(2)_{2}^{2+} \mathrm{O}_{6}^{2-} \text {, } \\
& \mathrm{La}_{2}^{3+} \mathrm{Cu}^{2+} \mathrm{O}_{4}^{2-} \quad \text { и } \quad \mathrm{Nd}_{2}^{3+} \mathrm{Cu}^{2+} \mathrm{O}_{4}^{2-} \text {. }
\end{aligned}
$$

Для сверхпроводящих оксидов выбор моделей основывался на данных [8]:

$$
\begin{aligned}
& \mathrm{La}_{1.85}^{3+} \mathrm{Sr}_{0.15}^{2+} \mathrm{Cu}^{2+} \mathrm{O}(1)_{2}^{2-} \mathrm{O}(2)_{2}^{1.925-}, \\
& \mathrm{Nd}_{1.85}^{3+} \mathrm{Ce}_{0.15}^{4+} \mathrm{Cu}^{1.85+} \mathrm{O}(1)_{2}^{2-} \mathrm{O}(2)_{2}^{2-}
\end{aligned}
$$

и

$$
\mathrm{Y}^{3+} \mathrm{Ba}_{2}^{1.98+} \mathrm{Cu}(1)^{2.04} \mathrm{Cu}(2)_{2}^{2.1+} \mathrm{O}(1)_{2}^{2.06-} \mathrm{O}(2)_{2}^{1.99-} \mathrm{O}(3)_{2}^{1.88-} \mathrm{O}(4)^{1.32} \text {. }
$$

Для всех узлов меди получено $0.55<V_{z z}<1.0$ (здесь и далее $V_{z z}$ приводится в единицах е/ $\left.\AA^{3}\right)$, а параметр асимметрии тензора решеточного ГЭП

$$
\eta=\left(V_{y y}-V_{x x}\right) / V_{z z}=0 .
$$

На рис. 3 показано соотношение между экспериментальными значениями $C_{\mathrm{Ni}}$ для центров ${ }^{61} \mathrm{Ni}^{2+}$ в узлах меди исследованных соединений и рассчитанными значениями главной компоненты тензора решеточного ГЭП $V_{z z}$ в тех же узлах. 


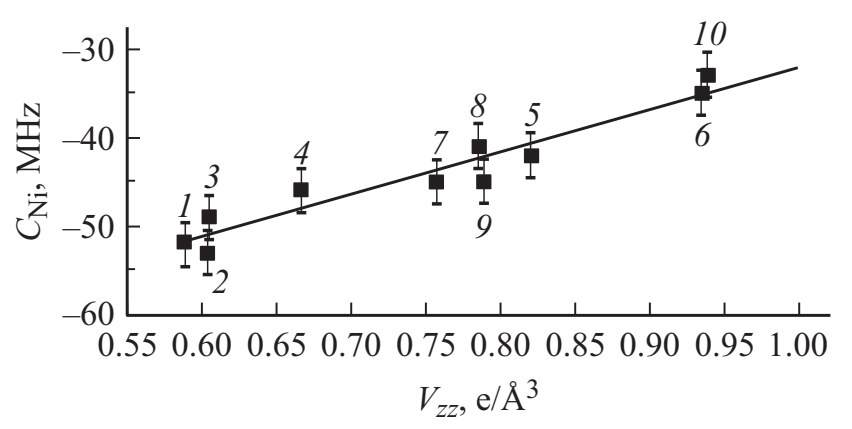

Рис. 3. Зависимость константы квадрупольного взаимодействия для центров ${ }^{61} \mathrm{Ni}$ в узлах меди $C_{\mathrm{Ni}}$ от главной компоненты тензора решеточного ГЭП в этих узлах $V_{z z}$. Обозначения узлов меди в решетках дано на рис. 2, а кроме того: $7-\mathrm{Sr}_{2} \mathrm{CuO}_{2} \mathrm{Cl}_{2}, 8-\mathrm{Ca}_{2} \mathrm{CuO}_{2} \mathrm{Cl}_{2}, 9-\mathrm{Nd}_{1.85} \mathrm{Ce}_{0.15} \mathrm{CuO}_{4}$, $10-\mathrm{Ca}_{0.85} \mathrm{Sr}_{0.15} \mathrm{CuO}_{2}$.

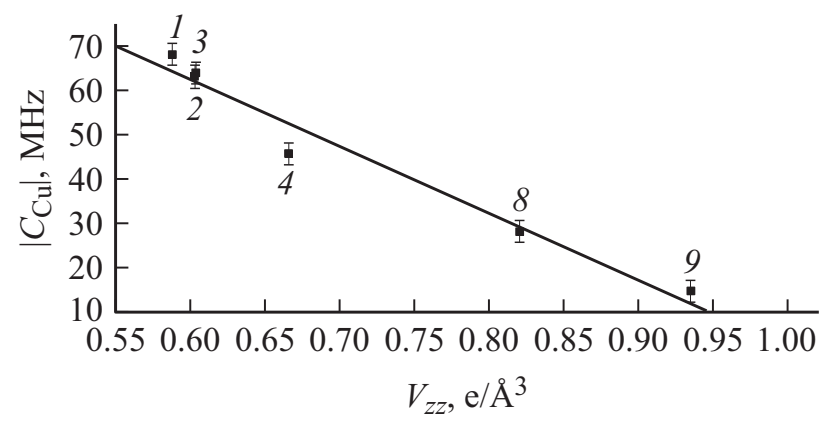

Рис. 4. Зависимость константы квадрупольного взаимодействия для центров ${ }^{63} \mathrm{Cu}$ в узлах меди от главной компоненты тензора решеточного ГЭП в этих узлах. Обозначения узлов меди в решетках дано в подписи к рис. 2 .

Зависимость на рис. 3 линейна и определяется соотношением

$$
C_{\mathrm{Ni}}=49 V_{z z}-81 \text {. }
$$

Из соотношения (3) следует, что линейная зависимость (5) является следствием одинаковой величины валентной составляющей в ГЭП для зонда $\mathrm{Ni}^{2+}$ в изученных металлоксидах двухвалентной меди. При этом уменьшение $\left|C_{\mathrm{Ni}}\right|$ при увеличении $V_{z z}$ свидетельствует о противоположных знаках валентного и решеточного вкладов в формуле (2) для центров $\mathrm{Ni}^{2+}$ и о выполнении соотношения $\left|(1-R) W_{z z}\right|>\left|(1-\gamma) V_{z z}\right|$ для центров $\mathrm{Ni}^{2+}$ в узлах меди. Поскольку измеренные значения $C_{\mathrm{Ni}}$ отрицательны, то с учетом $Q_{g r}^{61}>0$ для основного состояния ${ }^{61} \mathrm{Ni}$, величина $e Q_{g r}^{61}(1-R) W_{z z}$ для центров $\mathrm{Ni}^{2+}$ оказывается отрицательной и равной $-81(2) \mathrm{MHz}$.

На рис. 4 показана подобная рис. 3 зависимость константы квадрупольного взаимодействия $C_{\mathrm{Cu}}$ для центров ${ }^{63} \mathrm{Cu}^{2+}$ в узлах меди от рассчитанной выше главной компоненты тензора решеточного ГЭП в этих узлах. Как и на рис. 2, на рис. 4 приведены абсолютные величины $C_{\mathrm{Cu}}$. Видно, что зависимость на рис. 4 линейна и определяется соотношением

$$
\left|C_{\mathrm{Cu}}\right|=-150 V_{z z}+153,
$$

которое выполняется и для сверхпроводниковых, и для диэлектрических материалов.

Соотношение (3) означает, что линейная зависимость (6) является следствием неизменной величины валентной составляющей в ГЭП для зонда $\mathrm{Cu}^{2+}$ в металлоксидах двухвалентной меди. Уменьшение $\left|C_{\mathrm{Cu}}\right|$ с ростом $V_{z z}$ свидетельствует о противоположных знаках валентного и решеточного вкладов в формуле (3) и о выполнении соотношения $\left|(1-R) W_{z z}\right|>\left|(1-\gamma) V_{z z}\right|$ для центров $\mathrm{Cu}^{2+}$. Поскольку значения $V_{z z}>0$, то $W_{z z}<0$, а при $Q^{63}=-0.211 b[8]$ $e Q^{63}(1-R) W_{z z}=153(2) \mathrm{MHz}>0$. Таким образом, pacчеты ГЭП для узлов меди позволили установить знак констант квадрупольного взаимодействия для центров $\mathrm{Cu}^{2+}$, недоступный для прямого измерения [14-16]. Соотношение (6) можно записать без знака модуля: $C_{\mathrm{Cu}}=-150 V_{z z}+153$.

Главные значения валентного ГЭП $W_{z z}$ для обоих зондов $\mathrm{Cu}^{2+}$ и $\mathrm{Ni}^{2+}$ можно оценить, исходя из найденных валентных вкладов в константы квадрупольного взаимодействия $e Q(1-R) W_{z z}$ и известных квадрупольных моментов зондов. Если принять для $(1-R)$ типичное значение 0.7, то $W_{z z} \approx-30 \mathrm{e} / \AA^{3}$ и $W_{z z} \approx-20 \mathrm{e} / \AA^{3}$ для $\mathrm{Cu}^{2+}$ и $\mathrm{Ni}^{2+}$, соответственно. Их абсолютные значения и их примерное равенство для разных матриц, конечно, требуют квантово-механических расчетов, но отрицательные знаки можно объяснить в рамках модели кристаллического поля.

Во всех рассмотренных соединениях ближайшим окружением узлов меди является квадрат ионов $\mathrm{O}^{2-}[10-13]$. В этом случае расщепление в сильном кристаллическом поле приводит к тому, что наименьшую энергию имеет $d_{z^{2}}$-орбиталь, а наибольшую $d_{z^{2}-y^{2}}$-орбиталь. Электроны на этих орбиталях создают на ядре валентные ГЭП со значениями $W_{z z}=-\frac{4}{7} e\left\langle r^{-3}\right\rangle$ и $W_{z z}=\frac{4}{7} e\left\langle r^{-3}\right\rangle$, соответственно, где $\left\langle r^{-3}\right\rangle-$ среднее значение обратного куба радиуса $d$-орбитали. Ион $\mathrm{Ni}^{2+}$ имеет электронную конфигурацию $4 s^{2} 3 d^{6}$, в которой пять $d$-электронов образуют полузаполненную сферическую оболочку, а шестой попадает на нижний свободный $d_{z^{2}}$-уровень и создает $W_{z z}<0$. Ион $\mathrm{Cu}^{2+}$ имеет конфигурацию $3 d^{9}$, что соответствует дырке в замкнутой

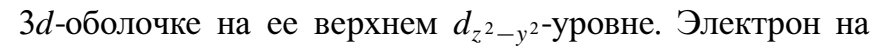
этом уровне создавал бы $W_{z z}>0$, а дырка создает $W_{z z}<0$.

\section{4. Выводы}

Эмиссионные мёссбауэровские спектры ${ }^{61} \mathrm{Cu}\left({ }^{61} \mathrm{Ni}\right)$ для диэлектрических металлоксидов двухвалентной меди $\mathrm{Ca}_{1-x} \mathrm{Sr}_{x} \mathrm{CuO}_{2}, \mathrm{Ca}_{2} \mathrm{CuO}_{2} \mathrm{Cl}_{2}, \mathrm{SrCuO}_{2}, \mathrm{Sr}_{2} \mathrm{CuO}_{2} \mathrm{Cl}_{2}$, $\mathrm{YBa}_{2} \mathrm{Cu}_{3} \mathrm{O}_{6}, \mathrm{La}_{2} \mathrm{CuO}_{4}$ и $\mathrm{Nd}_{2} \mathrm{CeCuO}_{4}$ отвечают квадрупольному и зеемановском взаимодействию ядер 
${ }^{61} \mathrm{Ni}$ с локальными полями в узлах меди, тогда как для сверхпроводящих металлоксидов $\mathrm{YBa}_{2} \mathrm{Cu}_{3} \mathrm{O}_{7}$, $\mathrm{La}_{1.85} \mathrm{Sr}_{0.15} \mathrm{CuO}_{4}$ и $\mathrm{Nd}_{1.85} \mathrm{Ce}_{0.15} \mathrm{CuO}_{4}$ спектры соответствуют взаимодействию квадрупольного момента ядер ${ }^{61} \mathrm{Ni}$ с тензором градиента электрического поля. Для сверхпроводящих и диэлектрических металлоксидов наблюдаются линейные зависимости постоянных квадрупольного взаимодействия как на ядрах ${ }^{61} \mathrm{Ni}$ (данные эмиссионной мёссбауэровской спектроскопии на изотопах $\left.{ }^{61} \mathrm{Cu}\left({ }^{61} \mathrm{Ni}\right)\right)$, так и на ядрах ${ }^{63} \mathrm{Cu}$ (данные ядерного магнитного резонанса на изотопе ${ }^{63} \mathrm{Cu}$ ) от расчетных значений главной компоненты тензора решеточного градиента электрического поля в узлах меди. Этот факт объясняется тем, что как для зондов ${ }^{61} \mathrm{Ni}^{2+}$, так и для зондов ${ }^{61} \mathrm{Cu}^{2+}$ валентная составляющая в суммарный тензор ГЭП в узлах меди не зависит от состава металлоксида.

\section{Благодарности}

Работа выполнена с использованием кристаллографического программного обеспечения Центра коллективного пользования „Материаловедение и диагностика в передовых технологиях“ (ФТИ им. А.Ф. Иоффе РАН).

\section{Конфликт интересов}

Авторы заявляют, что у них нет конфликта интересов.

\section{Список литературы}

[1] D. Farina, G. De Filippis, A.S. Mishchenko, N. Nagaosa, JhihAn Yang, D. Reznik, Th. Wolf, V. Cataudell. Phys. Rev. B 98, 121104 (2018).

[2] Shoji Yamamoto, Yusaku Noriki. Phys. Rev B 99, 094412 (2019).

[3] T. Miyamoto, Y. Matsui, T. Terashige, T. Morimoto, N. Sono, H. Yada, S. Ishihara, Y. Watanabe, S. Adachi, T. Ito, K. Oka, A. Sawa, H. Okamoto. Nature Commun. 9, 3948 (2018).

[4] A. Perucchi, P. Di Pietro, S. Lupi, R. Sopracase, A. Tebano, G. Giovannetti, F. Petocchi, M. Capone, D. Di Castro. Phys. Rev. B 97, 045114 (2018).

[5] Y. Zhong, S. Han, Y. Wang, Z. Luo, D. Zhang, L. Wang, W. Li, K. He, C.-L. Song, X.-C. Ma, Q.-K. Xue. Phys. Rev. B 97, 245420 (2018).

[6] G.Q. Liu, Q.B. Hao, H.L. Zheng, S.N. Zhang, X.Y. Xu, G.F. Jiao, L.J. Cui, P.F. Wang, C.S. Li. J. Phys. Conf. Ser. 1054, 01204230 (2018).

[7] J.C. Petersen, A. Farahani, D.G. Sahota, R. Liang, J.S. Dodge. Phys. Rev. B 96, 115133 (2017).

[8] N. Seregin, A. Marchenko, P. Seregin. Emission Mössbauer spectroscopy. Electron defects and Bose-condensation in crystal lattices of high-temperature supercomductors. Verlag: LAP LAMBERT. Academic Publishing GmbH \& Co. KG, Saarbrücken. $325 \mathrm{p}$.

[9] G.A. Bordovsky, A.V. Marchenko, A.V. Nikolaeva, P.P. Seregin, K.U. Bobokhuzhaev. Glass Phys. Chem. 41, 237 (2015).

[10] T. Siegrist, S.M. Zahurak, D.W. Murphy, R.S. Roth. Nature 334. 231 (1988).
[11] K. Yvon, M. Francois. Z. Phys. D-Condens. Matter 76, 413 (1989).

[12] X. Zhou, F. Wu, B. Yin, W. Liu, C. Dong, J. Li, W. Zhu, S. Jia, Y. Yao, Z. Zhao. Phys. C 233, 311 (1994).

[13] H. Haas, J.G. Correia. Hyperfine Interact. 176. 9 (2007).

[14] T. Takatsuka. K. Kumagai, H. Nakajima, A. Yamanaka. Physica C 185-189, 1071 (1991).

[15] Y. Yoshinari, H. Yasuoka, T. Shimizu, H. Takagi, Y. Tokura, S. Uchida. J. Phys. Soc. Jpn. 59, 36 (1990).

[16] T. Shimizu. J. Phys. Soc. Jpn. 62, 772 (1993).

Редактор Т.Н. Василевская 